\title{
Acceso oportuno y permanencia escolar de alumnos de 6 a 14 años en Sonora, 2003-2005
}

\author{
José Alfredo Flores Valdez*
}

Resumen: El concepto de calidad educativa se interpreta mejor a través del análisis de las diversas dimensiones que lo componen, en particular mediante juicios de valor acerca de la eficacia, eficiencia, pertinencia y equidad del sistema en su conjunto. Con este enfoque se han orientado varios estudios sobre la eficacia del sistema educativo basados en el análisis de los resultados académicos como sinónimo de calidad educativa, y han dejado de lado las concepciones que la relacionaban con el hecho de ofrecer acceso a la educación, como si este aspecto estuviera resuelto. Falta mucho por hacer, el análisis detallado de la cobertura en edad y grado permite apreciar avances significativos en Sonora de 2003 a 2005, y detectar con precisión las deficiencias principales. Así, se observan las mayores debilidades de acceso oportuno y permanencia, claves para el éxito escolar en la población de 10 a 12 y 14 años de edad, las etapas finales de educación primaria y secundaria. El reto es disminuir los índices de repetición en primaria, como medida decisiva y precautoria, para evitar otro problema grave en secundaria, la deserción.

Palabras clave: cobertura en edad y grado, acceso oportuno, permanencia, éxito escolar, calidad educativa, deserción.

Abstract: The concept of quality in education is better interpreted by analyzing its different dimensions, in particular by making judg-

\footnotetext{
* Director de Indicadores y Contexto Educativo, Instituto de Evaluación Educativa del Estado de Sonora (IEEES). Correspondencia: Olivares 38, colonia Villa Satélite, C.P. 83200. Hermosillo, Sonora, México. Teléfono (662) 260 7912. Correo electrónico: alfredo.flores@ieees.gob.mx
} 
ments about the efficiency, pertinence, and equity of the system as a whole. This approach has been used in various studies of the educational system based on the analysis of academic results, understood as synonymous of educational efficiency, ignoring the important and basic issue of access to education. There is much to be done; a detailed analysis of education coverage by age and grade not only shows meaningful progress in Sonora between 2003 and 2005, but also highlights the main problems. In this sense, the biggest weaknesses are timely access and permanence, the key to academic success in children aged 10-12 and 14 , who are in the final stages of elementary and middle school. The challenge is to avoid the repetition of school grades in elementary school as a preventive measure to avoid desertion, another big problem in middle school.

Key words: age and grade coverage, timely access, permanence, academic success, educational efficiency, desertion.

\section{Introducción}

Por décadas, la calidad del sistema educativo mexicano se ha medido por su capacidad de ofrecer su servicio a la población “demandante”. Las nociones actuales de calidad permiten reorientar el análisis hacia la capacidad de aprendizaje de la población como resultado del proceso de enseñanza formal. A escala internacional, el problema de la calidad en la educación, sobre todo en el nivel básico, ocupa el primer plano de la agenda educativa, y cobra mayor atención por parte de los actores políticos, sociales y económicos. El artículo cuarto de la Declaración Mundial sobre Educación para Todos de Jomtien, Tailandia (Organización de la Naciones Unidas para la Educación, la Ciencia y la Cultura, UNESCO 1990, 11) es más que elocuente al respecto, y permite apreciar la interacción de los componentes que conforman el concepto:

Que el incremento de las posibilidades de educación se traduzca en un desarrollo genuino del individuo y de la sociedad depende de que verdaderamente adquieran conocimientos útiles, capacidad de raciocinio, aptitudes y valores. De tal forma que la calidad educativa se vincula no 
sólo a los resultados de aprendizaje, sino también a las condiciones y a los procesos que conducen a ellos, así como al acceso, la igualdad y la equidad.

Según esta perspectiva, brindarles educación a todos en igualdad de condiciones permite reconocer y determinar el grado de desempeño de un sistema educativo, en lo referente a las oportunidades reales de la población para acceder y continuar recibiendo una educación de calidad. En América Latina se han establecido metas claras respecto a ofrecer un servicio educativo, como ejemplo, la Cumbre de las Américas planteó la necesidad de que en 2010,100 por ciento de los niños curse la educación básica y la concluya (Guadalupe 2002, 2), y así adquiera los conocimientos útiles, la capacidad de raciocinio y las aptitudes y valores que le permitan al individuo un desarrollo adecuado para enfrentar los cambios y escenarios futuros.

En fecha reciente, en México se ha establecido el compromiso de medir y mejorar la calidad educativa. Es por ello que el Programa Estatal de Educación 2004-2009 de Sonora, la establece en su primer eje y centro principal de todas sus acciones. En este sentido, se puede afirmar que así como la utopía de fines del siglo xIx fue universalizar la educación básica, base sobre la que se construyeron los sistemas educativos en América Latina, a lo largo del siglo pasado parece que se dieron las condiciones para el surgimiento de otra: brindar una educación de calidad en condiciones de equidad.

De esta manera, la capacidad del sistema educativo para ofrecer un servicio a quien lo demanda, pero sobre todo que sea de calidad, se transforma en un reto. En este escenario se requiere analizar un aspecto tan importante como el acceso equitativo en la igualdad de oportunidades, a través de la educación.

El estudio presente comprende el lapso de 2003 a 2005, como momento clave para evaluar la gestión educativa como efecto de la administración pública estatal actual, que abarca de 2003 a 2009; cubre tres ciclos escolares 2003-2004, 2004-2005 y 2005-2006.

\section{Cobertura en edad y grado, medida del acceso oportuno y permanencia en el sistema educativo \\ Cobertura bruta y neta}

Uno de los primeros indicadores utilizados para medir el funcionamiento del sistema educativo es la cobertura. Tradicionalmente se han definido dos 
indicadores para valorar la atención a la población demandante: cobertura bruta y cobertura neta. La primera se define como el porcentaje de alumnos atendidos (sin tomar en cuenta su edad) en un nivel educativo, con respecto al total de la población en la edad para cursarlo. Se calcula dividiendo a todos los alumnos inscritos en un nivel sobre los que, según su edad, se supone les corresponde cursarlo (tres a cinco en preescolar; 6 a 12 en primaria y 13 a 15 en secundaria). ${ }^{1}$

Para la cobertura neta, las autoridades educativas federales han determinado rangos de edad (normativa) según la permanencia ininterrumpida de los alumnos en cada grado, de tal manera que si uno ingresa a los tres años de edad a preescolar, culminará a los cinco y a los seis ingresará a primaria, y llegará a sexto a los 11, e ingresará a los 12 a secundaria, para terminarla a los 14 años. Este indicador se expresa como el porcentaje de alumnos, en edad normativa, inscritos al inicio del ciclo escolar en un nivel educativo, con respecto al total de la población en edad normativa del grado correspondiente. En este caso, tanto el numerador como el denominador tiene la misma base, por ejemplo, para la primaria se considerará la población inscrita de 6 a 11 años de edad, y se dividirá entre el total de la entidad de 6 a 11. El cuadro 1 sintetiza la edad normativa para la educación básica.

\section{Cuadro 1}

Edad normativa, en años, para cada tipo y nivel educativo

\begin{tabular}{|l|c|c|}
\hline Tipo & Nivel /tipo de educación & Edad normativa (años) \\
\hline Básico & Preescolar & 3 a 5 \\
& Primaria & 6 a 11 \\
& Secundaria & 12 a 14 \\
\hline
\end{tabular}

Fuente: elaboración propia.

A manera de ilustración, en el cuadro 2 se presentan los valores de ambos indicadores para los ciclos 2002-2003 y 2004-2005, en los cuales la cobertura neta de primaria fue superior a la bruta, debido a que su cálculo se basa en el mismo grupo de edad de la población (6 a 11 años), mientras que la

\footnotetext{
1 Para preescolar y secundaria se consideran los tres años de cada nivel, para calcular la edad en la que se supone la población debe cursar dicha etapa, sin embargo, para la primaria se estiman siete y no seis años como el periodo para su cálculo.
} 
bruta considera todavía en su denominador a la de 12 años, que en buena parte ya se encuentra inscrita en primero de secundaria (como se verá más adelante).

En el caso de secundaria, la relación entre cobertura bruta y neta es inversa, esta última presenta valores inferiores, básicamente por la compensación en los rangos de edad utilizados, mientras que el método de cálculo de la cobertura bruta es dividir al total de inscritos entre la población de 13 a 15 años. Entre ellos hay una proporción elevada de alumnos de 12, que están en primero de secundaria, y que son considerados en la base al denominador para este mismo indicador en primaria.

\section{Cuadro 2}

Cobertura bruta y neta en Sonora, ciclos 2002-2003 y 2004-2005

\begin{tabular}{|l|c|c|c|c|}
\hline \multirow{2}{*}{ Ciclo escolar } & \multicolumn{4}{|c|}{ Cobertura } \\
\cline { 2 - 5 } & \multicolumn{3}{|c|}{ Primaria } & \multicolumn{2}{c|}{ Secundaria } \\
\cline { 2 - 5 } & Bruta & Neta & Bruta & Neta \\
\hline $2002-2003$ & 91.8 & 100.3 & 85.5 & 76.1 \\
$2004-2005$ & 90.2 & 99.2 & 92.3 & 83.2 \\
\hline
\end{tabular}

Fuente: elaboración propia.

Ambos indicadores tienen limitaciones y su interpretación es delicada. En el caso de la cobertura bruta, su definición no distingue la edad de los alumnos inscritos, dentro de ésta se pueden encontrar los que ingresaron de forma tardía (mayores de seis años) y los que no obtuvieron los resultados que al final del ciclo escolar les permitieran proseguir, comúnmente conocidos como "repetidores". Para la cobertura neta, aunque es un indicador más preciso para determinar la participación educativa, ya que muestra la proporción de la población en edad de estudiar y que está matriculada, el manejo agregado por nivel educativo hace poco entendibles las tasas inferiores o superiores a 100 por ciento, ya que dichos valores no manifiestan un problema en la atención, debido al factor rezago o al hecho de que cierta población esté “adelantada”, que puede suceder dentro de un nivel educativo, por lo que esta medida no muestra si los estudiantes se encuentran o no en el grado y nivel correspondiente según su edad. Aun con el establecimiento de edades normativas, la inscripción adelantada, la reprobación, deserción o rezago educativo son factores que limitan la interpretación clara de estos indicadores. 
Las diferencias y deficiencias entre las coberturas bruta y neta dificultan el análisis del desempeño en la materia. No permiten determinar con exactitud el grado de rezago o avance en el acceso a la educación básica de la población, pero sí tener aproximaciones en la medición de la oferta del servicio educativo.

El presente análisis se realiza en dos dimensiones: la relativa al nivel educativo (primaria y secundaria) y la referente a las edades "idóneas” de atención de la población en dichos niveles, que es la base de este estudio.

Cobertura en edad y grado en Sonora 2003-2005

Como ya se planteó, el cálculo de las tasas actuales de cobertura pueden dar pie a interpretaciones erróneas, debido a que su variación no sólo es efecto de una mayor población inscrita, sino de otros indicadores como repetición, deserción o reingreso al sistema (como se explicará a continuación), por lo que dicha omisión puede perjudicar los objetivos y metas educativas.

Al respecto, la UNESCO, a través del Proyecto Regional de Indicadores Educativos en América Latina, estableció una serie de indicadores adicionales de cobertura, que dan cuenta del avance en la materia a través de la medición del alcance del servicio, según la edad de la población.

La cobertura en edad y grado explica el porcentaje de alumnos en edad normativa inscritos al inicio del ciclo escolar en el grado correspondiente, respecto al total de la población en edad para cursar dicho grado.

Un porcentaje cercano a 100 indicará que las escuelas no sólo satisfacen la demanda educativa, sino que lo hacen oportunamente. Este indicador mide la cobertura en el grado esperado de acuerdo a la edad y también permite observar la eficacia agregada del sistema, ya que da cuenta de la probabilidad real que tiene una cohorte de población de ingresar de manera oportuna y mantenerse así en el sistema. También indica el atraso escolar, fenómeno relevante en México, que repercute de modo crucial en las posibilidades reales de conclusión de los estudios.

El cuadro 3 presenta la distribución porcentual de la población inscrita según su edad en primaria y secundaria por grado educativo. ${ }^{2}$ La suma por columna determina la cobertura total para la población de esa edad, sin considerar el grado que cursa. En cada renglón se presenta la proporción de alumnos de cada edad, inscritos en alguno de los nueve grados de primaria y secundaria. La línea diagonal que forman los valores sombreados correspon-

\footnotetext{
2 Este desglose permite apreciar el avance o atraso de la población según su edad, los valores por encima del cien se pueden explicar debido a que el denominador utilizado para estos cálculos son estimaciones a mitad de año, así como a imprecisiones posibles al declarar la edad del alumno al momento de inscribirse.
} 
de a la proporción de la población, que según su edad normativa, debe cursar determinado grado.

\section{Cuadro 3}

Tasa de cobertura en edad y grado en primaria y secundaria, ciclo 2003-2004

\begin{tabular}{|c|c|c|c|c|c|c|c|c|c|}
\hline & \multicolumn{9}{|c|}{ Edades } \\
\hline Primaria & 6 & 7 & 8 & 9 & 10 & 11 & 12 & 13 & 14 \\
\hline Primero & 98.3 & 8.9 & 1.3 & 0.5 & 0.3 & 0.1 & 0.1 & 0.0 & 0.0 \\
\hline Segundo & 0.1 & 89.9 & 13.7 & 2.9 & 0.9 & 0.3 & 0.1 & 0.0 & 0.0 \\
\hline Tercero & 0.0 & 0.4 & 84.3 & 16.1 & 4.0 & 1.4 & 0.5 & 0.2 & 0.0 \\
\hline Cuarto & 0.0 & 0.0 & 0.5 & 80.5 & 17.3 & 5.1 & 1.5 & 0.4 & 0.1 \\
\hline Quinto & 0.0 & 0.0 & 0.0 & 0.4 & 77.1 & 18.4 & 5.6 & 1.8 & 0.4 \\
\hline Sexto & 0.0 & 0.0 & 0.0 & 0.0 & 0.7 & 76.6 & 17.6 & 5.4 & 1.3 \\
\hline Total en primaria & 98.4 & 99.2 & 99.8 & 100.3 & 100.3 & 101.9 & 25.4 & 7.9 & 1.8 \\
\hline \multicolumn{10}{|c|}{ Secundaria } \\
\hline \multicolumn{6}{|c|}{ Primero } & 1.1 & 71.0 & 18.1 & 5.2 \\
\hline \multicolumn{6}{|c|}{ Segundo } & 0.0 & 1.3 & 66.9 & 16.3 \\
\hline \multicolumn{6}{|c|}{ Tercero } & 0.0 & 0.0 & 2.3 & 55.1 \\
\hline \multicolumn{6}{|c|}{ Total en secundaria } & 1.1 & 72.3 & 87.4 & 76.6 \\
\hline \multicolumn{6}{|c|}{ Total (11-14 años) } & 103.0 & 97.8 & 95.2 & 78.4 \\
\hline
\end{tabular}

Fuente: elaboración propia, con información de Formato 911 de la Secretaría de Educación Pública (SEP).

Durante el ciclo 2003-2004, de los niños de seis años de edad en Sonora, la cobertura en primaria fue de 98.4 por ciento, donde prácticamente la totalidad (98.3) estaba en el grado correspondiente según su edad. Se puede observar cómo la cobertura total por edad aumenta hasta los 11 años (sexto de primaria), no obstante hay un deterioro constante en la atención oportuna de acuerdo a la edad normativa y grado escolar a partir de los siete años, por ejemplo, sólo 89.9 por ciento de los niños de esa edad cursaba segundo grado, mientras que del total de la población de diez años en Sonora 77.1 por ciento estaba inscrita en quinto.

El desglose de este indicador permite apreciar que 72.3 por ciento de los alumnos de 12 años ya estaba inscrito en primero de secundaria, mientras que la fórmula de cálculo para la cobertura bruta maneja como denominador base para primaria de 6 a 12 años, y para secundaria a los de 13 a 15, lo que explica las diferencias entre los valores de ambas coberturas.

Las mayores dificultades de atención oportuna (edad normativa y grado escolar respectivo) se observaron en los jóvenes de 14 años; sólo cinco de 
cada diez que deberían haber estado culminando la educación básica, se encontraban inscritos en tercero.

El cuadro 4 muestra la cobertura en edad y grado para el ciclo 20052006. El sistema mejoró su capacidad de atención oportuna en todos los grados (cifras sombreadas); por ejemplo, pasó de 76.6 a 80.0 por ciento en la población de 11 años (sexto de primaria) y de 77.1 a 83.0 en la de diez. El mayor incremento se dio en la atención de los jóvenes de 14 años, al pasar de 55.1 en 2003-2004 a 65.3 en 2005-2006; un aumento de poco más de 5 mil jóvenes atendidos oportunamente.

\section{Cuadro 4}

Tasa de cobertura en edad y grado en primaria y secundaria, ciclo 2005-2006

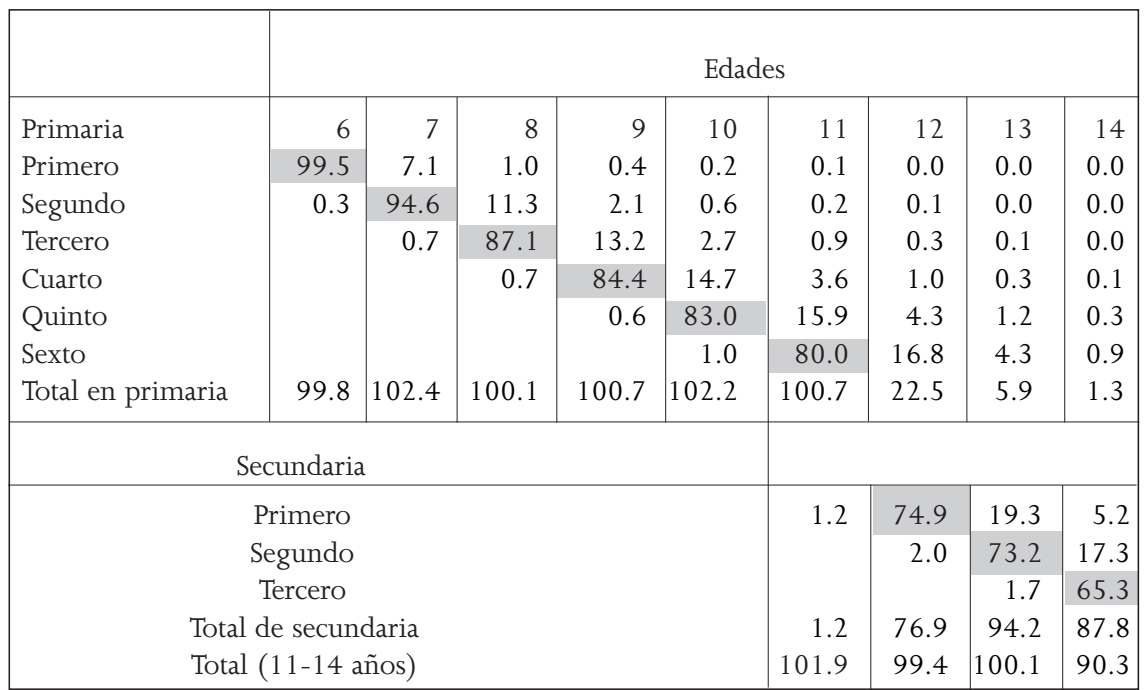

Fuente: Flores Valdez y Barrientos (2007).

Sin embargo, la brecha que separa la atención oportuna entre la población de siete y ocho años se acrecentó, mientras en 2003-2004 la diferencia era de 5.6 puntos porcentuales para 2005-2006 fue de 7.1.

A través de este cálculo, se han identificado tres grados de cobertura según el momento en que se atiende a la población: a) la que determina la proporción de la población, que según su edad le corresponde cursar determinado grado (llamada “oportuna”) — representada por los valores sombreados- - b) la proporción de una edad determinada, que cursa un grado inferior al normativo, denominada "tardía” y c) al porcentaje de la adelantada, es decir, que de acuerdo a su edad debiera estar en cierto grado, pero 
cursa el siguiente, conocida como "prematura". De la diferencia entre la suma de éstas y el total resulta la "población no atendida”.

\section{Gráfica 1}

Cobertura tardía, 2003-2005 de la educación primaria y secundaria en Sonora

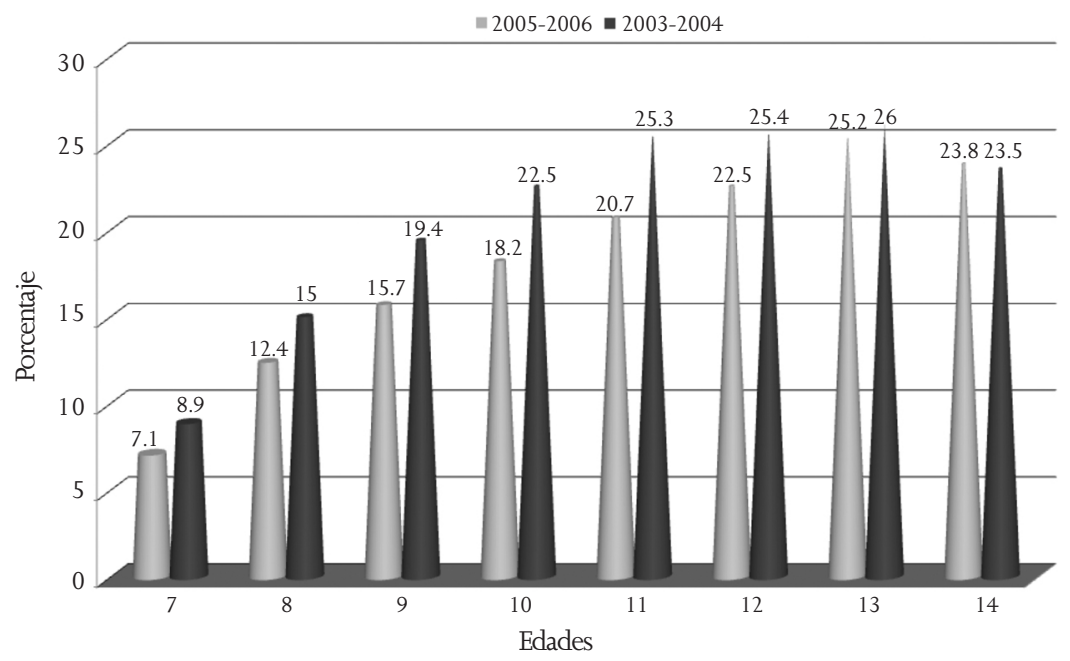

Fuente: elaboración propia, con base en la información de los cuadros 3 y 4.

Como complemento para los cuadros 3 y 4, la gráfica 1 presenta la evolución de la cobertura tardía al ciclo escolar 2005-2006, partiendo del 2003-2004. No se incluye la población no atendida, ya que en la mayoría de las edades, la cobertura total prácticamente fue de 100 por ciento.

Es notorio el aumento en la cobertura oportuna y la disminución en la tardía, para el ciclo escolar 2005-2006, que muestra una mejoría en las prácticas educativas, rendimiento escolar de los alumnos, supervisión, empeño de los estudiantes, en general una serie de factores que se reflejan en menor reprobación o repetición en cada escuela, lo que permite a los estudiantes mejorar el tránsito oportuno entre los diferentes grados.

De aquí se desprende que el sistema ha garantizado el acceso "oportuno" a todos los niños de seis años de edad (primaria), y ha mejorado sustancialmente la atención oportuna, no obstante las dificultades en la permanencia o tránsito ininterrumpido de los alumnos, sobre todo de secundaria y en particular en los de 14 años, entre quienes la cobertura tardía aumentó, y pasó de 23.5 a 23.8 por ciento de 2003 a 2005. 
Problemas principales de la conservación de la cobertura oportuna

En esta sección se explicarán las causas de las variaciones observadas arriba, a través del análisis de fuentes secundarias y la construcción de un índice de conservación de la cobertura oportuna. Por medio de un coeficiente que mide su avance o retroceso, se comparó el valor de la cobertura en edad para G+1 con la de edad para G (en este caso el ciclo 2005-2006 con el 20032004, dividiendo el primero sobre el segundo), de tal manera que un valor 0 indica una pérdida completa, mientras que 1, una conservación total de la cobertura del punto de partida, como se refleja en el cuadro 5. Esto permite determinar la edad en que ocurren los problemas mayores de retención de la población, con lo que se detectan las etapas clave de atención.

Como se observa en el cuadro 5, el sistema ha sido capaz de conservar oportunamente, en 2005 a 88.6 por ciento de la población de seis años inscrita en 2003, a partir de la edad de nueve se advirtió un incremento gradual de 93.9 hasta 99.4 por ciento de los alumnos de 11 años (sexto de primaria), significa que el sistema conservó de manera oportuna, a través de dos ciclos escolares, prácticamente a todos los estudiantes de siete a nueve años de edad.

La tendencia en secundaria es inversa, aquí se conservó, en el ciclo 20052006, a 97.1 por ciento que estaba atendido de acuerdo a su edad en quinto de primaria en el ciclo 2003-2004, en cuanto al segundo y tercero de secundaria sólo se conservó 95.6 y 92.0 por ciento, respectivamente.

De la misma forma, el cuadro 5 presenta los grupos de edad donde ocurre la mayor caída en la cobertura oportuna, y que se puede explicar de acuerdo a sus componentes; la columna cinco muestra su tasa de variación, mientras que la seis y siete desglosan sus causas. La mayor caída ocurrió entre la población de seis a ocho (11.39 por ciento), 12 a 14 (8.03) y siete a nueve años de edad (6.12). Las causas principales fueron:

- Repetición: en la población de seis a ocho años fue de 13.79 por ciento, mientras que del grupo de siete a nueve fue de 9.12. No existió deserción, al contrario, se incorporaron 2.4 y 3.0 por ciento de niños en ese rango de edad, respectivamente.

- Deserción: 30.5 por ciento entre el grupo de 12 a 14 años. La disminución de la repetición fue de 22.4 por ciento, con esto se concluye que los repetidores fueron quienes desertaron.

La gráfica 2 presenta la conservación del coeficiente de cobertura oportuna (CCOP) para los ciclos 2004-2005 y 2005-2006, se aprecia un mejoramiento donde destaca la reincorporación de niños entre los 10 y 11 años. 


\section{Cuadro 5}

Conservación de la cobertura oportuna en los ciclos escolares: 2003-2004 y 2005-2006, en Sonora

\begin{tabular}{|c|c|c|c|c|c|c|}
\hline \multirow[b]{2}{*}{ 1.Edad } & \multirow[b]{2}{*}{$\begin{array}{l}\text { 2. Cobertura } \\
\text { oportuna } \\
\text { 2003-2004 } \\
(\%)\end{array}$} & \multirow[b]{2}{*}{$\begin{array}{l}\text { 3. Cobertura } \\
\text { oportuna } \\
\text { 2005-2006 } \\
\text { (\%) }\end{array}$} & \multicolumn{2}{|c|}{$\begin{array}{l}\text { Conservación de la } \\
\text { cobertura oportuna }\end{array}$} & \multicolumn{2}{|c|}{$\begin{array}{c}\text { Explicación de la } \\
\text { variación }\end{array}$} \\
\hline & & & 4. Coeficiente & $\begin{array}{l}\text { 5. Variación } \\
(\%)\end{array}$ & $\begin{array}{l}\text { 6. Variación } \\
\text { de la } \\
\text { cobertura } \\
\text { total }(\%)^{1}\end{array}$ & $\begin{array}{c}\text { 7.Variación } \\
\text { del atraso } \\
\text { escolar } \\
(\%)^{2}\end{array}$ \\
\hline 6 & 98.3 & 99.5 & & & & \\
\hline 7 & 89.9 & 94.6 & & & & \\
\hline 8 & 84.3 & 87.1 & 0.886 & -11.39 & 2.4 & -13.79 \\
\hline 9 & 80.5 & 84.4 & 0.939 & -6.12 & 3.0 & -9.12 \\
\hline 10 & 77.1 & 83.0 & 0.985 & -1.54 & 2.1 & -3.64 \\
\hline 11 & 76.6 & 80.0 & 0.994 & -0.62 & -0.9 & 0.28 \\
\hline 12 & 71.0 & 74.9 & 0.971 & -2.85 & -1.2 & -1.65 \\
\hline 13 & 66.9 & 73.2 & 0.956 & -4.44 & -11.4 & 6.96 \\
\hline 14 & 55.1 & 65.3 & 0.920 & -8.03 & -30.5 & 22.47 \\
\hline
\end{tabular}

${ }^{1}$ Variación porcentual entre la cobertura total de dos ciclos, para dos edades continuas; los resultados con signo negativo denotan deserción, y los de positivo reingreso de la población en esa edad.

${ }^{2}$ Tasa de variación de la cobertura oportuna menos caída de la cobertura, un resultado negativo indica repetición, mientras que uno positivo señala una recuperación en esa edad.

Fuente: elaboración propia, con base en los cuadros 3 y 4, tomado de Guadalupe $(2002,15)$.

\section{Gráfica 2}

Evolución del coeficiente de cobertura oportuna en primaria y secundaria

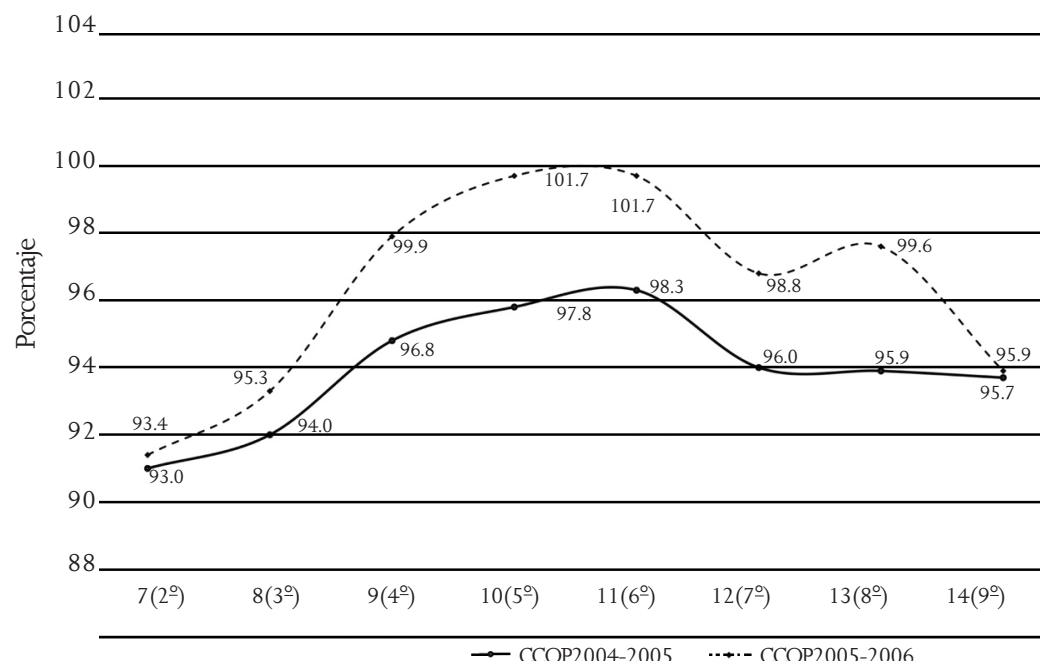

Fuente: elaboración propia. 
En consecuencia, los problemas que explican la caída en la cobertura oportuna en ambos niveles son diferentes; para los estudiantes de primaria la causa principal fue el incremento en la tasa de repetición, mientras que para los de secundaria fue el aumento de la deserción.

\section{Conclusiones}

El Sistema Educativo Estatal ha mejorado la atención a los niños y jóvenes en edad escolar básica en el periodo analizado, sobre todo entre los grupos de edad de seis y siete y 12 a 14 años. Sólo la atención oportuna a los de 14 se incrementó a una tasa de 10 por ciento en promedio anual (la de mayor aumento). Por lo que, para 2008 se tendría que haber atendido de manera oportuna a 71.6 por ciento de la población de 14 años en tercero de secundaria.

Si bien los avances en la entidad en este periodo han sido importantes, la meta de garantizar el acceso, pero sobre todo la conclusión de 100 por ciento de los alumnos de educación básica para 2010 será un reto difícil de cumplir. Al igual que a escala nacional (INEE 2006, 4), en Sonora, a partir de los 12 años de edad se observan incrementos notables en el número de jóvenes fuera de la escuela.

En la entidad hay dos etapas de desarrollo en lo concerniente a la cobertura según el nivel educativo; por una parte, prácticamente toda la población en edad de ingresar a primaria lo hace de manera oportuna, con ligeras dificultades en la conservación o permanencia entre los 10 y 11 años de edad (últimos grados), y el problema mayor es la repetición. Por otra parte, en la secundaria hay más dificultades para garantizar el acceso oportuno y sobre todo la permanencia de sus estudiantes, que se agudizan en los alumnos de 14 años (tercero), en particular por el abandono (deserción).

En este sentido, en México se recomienda que cada entidad analice la situación desagregada (por municipio, localidad, zona escolar, etcétera), para focalizar esfuerzos, lo que permitirá identificar a los lugares con la atención mínima.

El reto más importante para Sonora, conforme a lo analizado, es mantener a la población de 10 a 12 años dentro del sistema educativo en los ciclos escolares subsecuentes y disminuir la repetición, factor que en apariencia termina por desalentar a los estudiantes en los niveles siguientes, propiciando la deserción. Lo anterior coincide con la obligatoriedad nacional de realizar un esfuerzo mayor para garantizar el acceso a la secundaria, y asegurar 
la permanencia de los alumnos hasta que la concluyan (Instituto Nacional para la Evaluación Educativa, INEE 2005a, 4).

Para universalizar el acceso oportuno a la educación, como base del derecho equitativo a una educación de calidad, es necesario:

- Atender, a través de políticas sectoriales, los problemas económicos, familiares, geográficos e institucionales, que impiden que los niños asistan a la escuela

- Disminuir la cobertura tardía, a través de estrategias que reduzcan o eviten la reprobación y el atraso escolar dentro de la escuela, en particular entre primero y segundo año de primaria, y segundo y tercero de secundaria

- Crear e implementar estrategias de apoyo y orientación hacia los alumnos y padres de familia sobre la importancia de continuar en la escuela, dirigidas en específico a los estudiantes que están por concluir sus estudios, de los 10 a 11 y 13 a 14 años de edad

- Identificar en el ámbito municipal a las niñas y niños de 8 a 14 años que no van a la escuela, para lograr que ingresen o regresen

- Identificar a los alumnos en riesgo de abandonar sus estudios, para analizar las causas posibles de su condición de abandono y elaborar estrategias para disminuirlas.

Esta propuesta metodológica internacional se retoma para Sonora, con el fin de atender preocupaciones clave de política educativa como el acceso, permanencia y conclusión, que a su vez permitirá identificar los grados y las edades de mayor atraso escolar. La réplica de esta metodología a escala municipal o local permitirá reconocer los problemas principales.

Con este trabajo se confirma la necesidad de utilizar indicadores más directos para medir la cobertura, y dejar de lado los de "participación" (Guadalupe 2002, 18), así como reconsiderar los intervalos de edades normativas para cursar la educación básica (INEE 2006, 4), debido a la incorporación de diferentes niveles a la obligatoriedad del sistema educativo.

No obstante, también hay que aclarar que este indicador directo es sensible a las variaciones entre la edad cumplida y el grado escolar (Ibid., 285), aunado a que para su cálculo continuo se recurre a estimaciones de la población. Y los momentos más oportunos para su valoración estatal, municipal, por localidad o zona escolar estarán “acotados” por los instantes de levantamiento de información censal. 


\section{Bibliografía}

Flores Valdez, José Alfredo y Soledad L. Barrientos. 2007. Sistema de Indicadores Educativos del Estado de Sonora. Hermosillo: IEEES.

Gobierno del Estado de Sonora. 2004. Programa Estatal de Educación 2004-2009. Educación para competir y progresar. Hermosillo: Secretaría de Educación y Cultura.

Guadalupe M., César. 2002. Indicadores de cobertura, eficiencia y flujo escolar: necesidades de política, problemas metodológicos y una propuesta. Oficina Regional de Educación para América Latina y el Caribe, http://www.unesco.cl/biblioteca/documentos/indicadores_de_cobertura (20 de junio de 2006).

INEE. 2006. Evaluación del acceso y la permanencia de alumnos en el sistema educativo: un seguimiento desde los tres hasta los 17 años. Desafíos Educativos 5.

2006a. Panorama educativo de México 2005. México: INEE.

2005. PISA para docentes. La evaluación como oportunidad de aprendizaje. México: SEP.

2005a. Mejorar la cobertura en educación básica. Desafíos Educativos 1 .

SEP. 2004. Formulario de indicadores educativos. México: Dirección General de Planeación, Programación y Presupuesto de la SEP.

UNESCO. 1990. Declaración mundial sobre educación para todos y Marco para satisfacer las necesidades básicas de aprendizaje. http://www.oei. es/quipu/marco_jomtien.pdf (30 de agosto de 2007). 